typeset using JPSJ.sty $<$ ver.1.0b $>$

\title{
Neutron Scattering Study of Temperature-Concentration Phase Diagram of $\left(\mathrm{Cu}_{1-x} \mathrm{Mg}_{x}\right) \mathrm{GeO}_{3}$
}

\author{
Hironori Nakad , Masakazu Nishi, Yasuhiko FujiI, Takatsugu MasudA ${ }^{1}$, \\ Ichiro Tsukada ${ }^{1, * *}$, Kunimitsu Uchinokura ${ }^{1}$, Kazuma Hirota ${ }^{2}$, and Gen Shirane ${ }^{3}$ \\ Neutron Scattering Laboratory, Institute for Solid State Physics, University of Tokyo, 106-1 Shirakata, Tokai, \\ Ibaraki 319-1106. \\ ${ }^{1}$ Department of Applied Physics, University of Tokyo, 7-3-1 Hongo, Bunkyo-ku, Tokyo 113-8656. \\ ${ }^{2}$ Department of Physics, Tohoku University, Sendai 980-8578. \\ ${ }^{3}$ Department of Physics, Brookhaven National Laboratory, Upton, NY 11973-5000, USA.
}

(Received April 4, 2018)

\begin{abstract}
In doped $\mathrm{CuGeO}_{3}$ systems, such as $\left(\mathrm{Cu}_{1-x} \mathrm{Zn}_{x}\right) \mathrm{GeO}_{3}$ and $\mathrm{Cu}\left(\mathrm{Ge}_{1-x} \mathrm{Si}_{x}\right) \mathrm{O}_{3}$, the spin-Peierls (SP) ordering $\left(T \leq T_{S P}\right)$ coexists with the antiferromagnetic $(\mathrm{AF})$ phase $\left(T \leq T_{N} \leq T_{S P}\right)$. $T_{S P}$ decreases while $T_{N}$ increases with increasing $x$ in low doping region. For higher $x$, however, the SP state disappears and only the AF state remains. These features are common for all the doped $\mathrm{CuGeO}_{3}$ systems so far studied, indicating the existence of universal $T-x$ phase diagram. Recently, Masuda et al. carried out comprehensive magnetic susceptibility $(\chi)$ measurements of $\left(\mathrm{Cu}_{1-x} \mathrm{Mg}_{x}\right) \mathrm{GeO}_{3}$, in which doping concentration can be controlled significantly better than the $\mathrm{Zn}$ doped systems. They found that $T_{N}$ suddenly jumps from 3.43 to $3.98 \mathrm{~K}$ at the critical concentration $x_{c} \sim 0.023$ and that a drop in $\chi$ corresponding to the SP ordering also disappears at $x>x_{c}$. They thus concluded that there is a compositional phase boundary between two distinct magnetic phases. To clarify the nature of two phases, we performed neutron-scattering measurements on $\left(\mathrm{Cu}_{1-x} \mathrm{Mg}_{x}\right) \mathrm{GeO}_{3}$ single crystals with various $x$. Analysis of the data at fixed temperature points as a function of doping concentration has revealed sudden changes of order parameters at the critical concentration $x_{c}=0.027 \pm 0.001$. At finite temperatures below $T_{N}$, the drastic increase of the AF moment takes place at $x_{c}$. The spin-Peierls order parameter $\delta$ associated with lattice dimerization shows a precipitous decrease at all temperature below $T_{S P}$. However, it goes to zero above $x_{c}$ only at the low temperature limit.
\end{abstract}

KEYWORDS: $\left(\mathrm{Cu}_{1-x} \mathrm{Mg}_{x}\right) \mathrm{GeO}_{3}$, spin-Peierls transition, antiferromagnetic transition, phase diagram, neutron scattering

\footnotetext{
* Present address: Photon Factory, Institute of Materials Structure Science, High Energy Accelerator Research Organization, Tsukuba, Ibaraki 305-0801. E-mail: hironori.nakao@kek.jp

** Present address: Central Research Institute of Electric Power Industry, 2-11-1 Iwado Kita, Komae-shi, Tokyo $201-8511$.
} 


\section{$\S 1 . \quad$ INTRODUCTION}

Since the first inorganic spin-Peierls(SP) compound $\mathrm{CuGeO}_{3}$ was discovered, 1 t extensive studies have been performed to understand the mechanism of the SP transition. In the course of studying doping effects on the SP state using $\mathrm{Cu}\left(\mathrm{Ge}_{1-x} \mathrm{Si}_{x}\right) \mathrm{O}_{3}$, Regnault et al. 1 . found that the spin-Peierls (SP) state $\left(T \leq T_{S P}\right)$ coexists with the antiferromagnetic $(\mathrm{AF})$ state $\left(T \leq T_{N} \leq T_{S P}\right)$. The discovery of coexistence has attracted much interest because it is a novel spin system for studying competition and cooperation between a singlet state with lattice dimerization and a three-dimensional Néel state. Fukuyama et al. .3) have theoretically shown that impurity-induced moments and lattice dimerization are both true long-range orderings though they have spatial modulations reflecting distribution of impurities. Kojima et al. (- observed that antiferromagnetic ordering is indeed spatially modulated by muon spin relaxation $(\mu \mathrm{SR})$.

Two classes of doped systems are so far reported, i.e. the site-random system such as $\left(\mathrm{Cu}_{1-x} M_{x}\right) \mathrm{GeO}_{3} \quad(M=\mathrm{Zn}$, 6.6 $\mathrm{Mg}$.96) and the bond-random system such as $\left.\mathrm{Cu}\left(\mathrm{Ge}_{1-x} \mathrm{Si}_{x}\right) \mathrm{O}_{3} .10,11,12\right)$ Both systems have very similar temperature vs. doping-concentration $T-x$ phase diagrams. $T_{S P}$ decreases almost linearly from $14.2 \mathrm{~K}$ at $x=0$ with increasing $x$. The SP order parameter, i.e. lattice dimerization $\delta$, also decreases as $x$ increases, and finally disappears at higher $x$. On the other hand, the AF order takes place at $T_{\mathrm{N}}=28.5 \mathrm{mK}$ even at $x=0.00112$,

and gradually increases with increasing $x$. $T_{N}$ reaches the maximum temperature and disappears at the high concentration region.

Martin et al. 1 carried out comprehensive neutron scattering experiments on $\mathrm{Zn}$-doped $\mathrm{CuGeO}_{3}$. They reported that the SP phase exists in samples with exceptionally high concentrations of Zn. They also pointed out that it is hard to dope Zn uniformly, particularly at high concentrations. Masuda et al. 9 reported that the doping concentration can be controlled significantly better in $\left(\mathrm{Cu}_{1-x} \mathrm{Mg}_{x}\right) \mathrm{GeO}_{3}$, and carried out comprehensive magnetic susceptibility $(\chi)$ measurements on high quality single crystals of $\left(\mathrm{Cu}_{1-x} \mathrm{Mg}_{x}\right) \mathrm{GeO}_{3}$ to establish the $T-x$ phase diagram. At $x=0.023$, they found two anomalies at $T=3.43$ and $3.98 \mathrm{~K}$, which they ascribed to AF orderings, while another anomaly at $T=10 \mathrm{~K}$ clearly indicates the $\mathrm{SP}$ transition, as shown in Fig. 1 . They

Fig. 1. (Bottom) The temperature-concentration phase diagram of $\left(\mathrm{Cu}_{1-x} \mathrm{Mg}_{x}\right) \mathrm{GeO}_{3}$ previously reported by magnetic susceptibility measurements [ref. 9]. At $x<x_{c}$ the AF ordering is realized on a dimerized lattice (D-AF) while the AF ordering is on a uniform lattice (U-AF) at $x>x_{c}$. (Top) Raw data of susceptibility measured on the $x=0.023$. Two $T_{N}$ 's suggest coexistence of D-AF and U-AF phases near $x_{c}$.

reported that the $x$ dependence of $T_{N}$ clearly exhibits a discontinuity at this concentration and a 
drop in $\chi$ corresponding to the SP ordering also disappears at $x>0.023$. From these results, they concluded that there exists a compositional phase boundary at critical concentration $x_{c}=0.023$ between two different magnetic phases. At $x<x_{c}$, the AF ordering is realized on a dimerized lattice (D-AF) while the $\mathrm{AF}$ ordering on a uniform lattice (U-AF) at $x>x_{c}$.

To understand the nature of possible compositional transition and two different phases, we have performed neutron-scattering measurements on $\left(\mathrm{Cu}_{1-x} \mathrm{Mg}_{x}\right) \mathrm{GeO}_{3}$ single crystals with various $x$ values. We indeed confirmed that the SP order parameter, i.e. lattice dimerization $\delta$, exhibits a discontinuous change at $x_{c}$ below $T_{S P}$, which is clearly seen at low temperatures in particular. We also found that the AF ordered moment at $1.3 \mathrm{~K}$ jumps. For $x \geq x_{c}$, however, the SP order parameter $\delta$ remains finite at finite temperatures though $\delta$ approaches zero at $T=0 \mathrm{~K}$. The present results support the existence of compositional phase boundary at $x_{c}$ and the disappearance of SP ordering for $x>x_{c}$ at $T=0 \mathrm{~K}$, though the SP phase at finite temperature survives in some $T-x$ region above $x_{c}$.

\section{§2. EXPERIMENTAL DETAILS}

A series of high-quality $\left(\mathrm{Cu}_{1-x} \mathrm{Mg}_{x}\right) \mathrm{GeO}_{3}$ single crystals $(x=0.017,0.026,0.028,0.032,0.041$, 0.082) used in the present experiment were grown by a floating-zone method.9) A typical size of crystal was $20 \times 5 \times 3 \mathrm{~mm}^{3}$, in which the longest direction was parallel to the orthorhombic $c$-axis. The concentration of $\mathrm{Mg}$ dopant, $x$, was carefully determined by an inductively coupled plasma atomic emission spectroscopy (ICP-AES), in which error is typically within \pm 0.001 . 9 )

Neutron-scattering experiments were carried out on ISSP-owned triple-axis spectrometers installed at the JRR-3M reactor of Japan Atomic Energy Research Institute. A crystal was mounted in an aluminum can with He exchange gas, which was attached to a cold finger of an Orange cryostat capable of reaching $1.3 \mathrm{~K}$. The sample was aligned so as to place the $(h k h)$ or $(0 k l)$ zone in scattering plane. Incident neutron beams were monochromatized with the $\left(\begin{array}{lll}0 & 0 & 2\end{array}\right)$ reflection of pyrolytic graphite (PG) to select $14.7 \mathrm{meV}$ or $5 \mathrm{meV}$ and the $\mathrm{PG}\left(\begin{array}{lll}0 & 0 & 2\end{array}\right)$ reflection was also used for analyzing neutron energy. A double PG or Be filter was used to reduce higher-order contaminations. The beams were horizontally collimated, with a typical configuration of 40'-40'-sample-40'-80' in sequence from the neutron source (reactor) to the detector.

The present $T-x$ phase diagram consists of traces of $T_{S P}(T, x)$ and $T_{N}(T, x)$, which were determined at each $x$ using temperature dependence of superlattice reflections $I_{S P}\left(\frac{3}{2} 1 \frac{3}{2}\right)$ and $I_{A F}\left(\begin{array}{lll}0 & 1 & \frac{1}{2}\end{array}\right)$, respectively. Effective atomic displacement $\delta_{\text {eff }}$ for lattice dimerization in the SP state and effective magnetic moment $\mu_{e f f}$ in the AF state were evaluated by comparing several

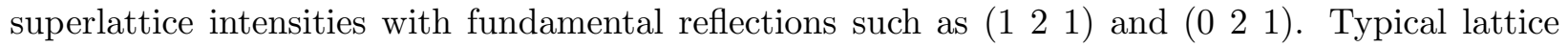
constants at room temperature are $\mathrm{a}=4.87 \AA, \mathrm{b}=8.49 \AA$, and $\mathrm{c}=2.95 \AA$ for $x=0.032$.

\section{§3. EXPERIMENTAL RESULTS}




\subsection{Phase transition}

Comprehensive neutron-scattering studies were carried out using a series of $\left(\mathrm{Cu}_{1-x} \mathrm{Mg}_{x}\right) \mathrm{GeO}_{3}$ single crystals in a wide concentration range $(x=0.017-0.082)$. For all the samples, we measured temperature dependence of both the $\mathrm{SP}$ and $\mathrm{AF}$ superlattice reflections, $I_{S P}\left(\frac{3}{2} 1 \frac{3}{2}\right)$ and $I_{A F}\left(01 \frac{1}{2}\right)$. The magnetic susceptibilities were also measured for comparison. Typical results are shown for $x=0.017$ and 0.032 in Figs. 2 and 3, respectively.

For the sample with $x=0.017$, which is below the critical concentration $x_{c}$, two sharp anomalies are

Fig. 2. Temperature dependence of the magnetic susceptibility (top) and the neutron intensities of the superlattice peaks of $I_{S P}\left(\begin{array}{lll}\frac{3}{2} & 1 & \frac{3}{2}\end{array}\right)$ and $I_{A F}\left(\begin{array}{lll}0 & 1 & \frac{1}{2}\end{array}\right)$ corresponding to the SP and AF phases, respectively, (bottom) for the sample with $x=0.017<x_{c}$. Both measurements clearly show $T_{S P}$ and $T_{N}$.

Fig. 3. Temperature dependence of the magnetic susceptibility (top) and the neutron intensities of superlattice peaks of $I_{S P}\left(\frac{3}{2} 1 \frac{3}{2}\right)$ and $I_{A F}\left(\begin{array}{lll}0 & 1 & \frac{1}{2}\end{array}\right)$ corresponding to the SP and AF phases, respectively, (bottom) for the sample with $x=0.032>x_{c}$. The susceptibility measurement shows no sign of $T_{S P}$ while neutron scattering data clearly show a superlattice peak and a well-defined $T_{S P}$.

seen in the susceptibility. These temperatures are in good agreement with $T_{N}$ and $T_{S P}$ determined from the order parameters. In the $x=0.032$ sample, which is above $x_{c}$, the AF transition is observed in both the susceptibility and neutron-scattering measurements. However, the SP transition is not noticeable in the susceptibility data though the neutron scattering result clearly shows the SP ordering. Note that it is already difficult at $x=0.023$ to define $T_{S P}$ by susceptibility measurement as shown in Fig. 1. Although Masuda et al.9) concluded that the SP phase completely vanishes at $x>x_{c}$ from their susceptibility measurements, the present neutron-scattering study unambiguously demonstrates that the SP phase survives in that region. As we show later, however, the SP phase at $x>x_{c}$ exhibits significant reduction in the order parameter, broadening of the transition temperature, and decrease of the correlation length, all of which indicate that there indeed exists a compositional phase boundary at $x_{c}$ as Masuda et al. (9) $^{\text {(1) }}$ pointed out.

In order to quantitatively evaluate transitions at $T_{S P}$ and $T_{N}$, neutron-scattering data were least-square-fitted with the following equation: 


$$
\begin{gathered}
I(T)=I_{0}+\frac{I}{\sqrt{\pi} \Delta T} \int_{T}^{\infty}\left(\frac{\tau-T}{\tau}\right)^{2 \beta} \exp \left[-\left(\frac{\tau-T_{C}}{\Delta T}\right)^{2}\right] d \tau \\
\left(T_{C}=T_{S P} \text { or } T_{N}\right)
\end{gathered}
$$

We have assumed a power-law description with a Gaussian distribution of transition temperature as previously employed for a pure $\mathrm{CuGeO}_{3} .13$. Tables I and II summarize the fitting parameters thus obtained for various concentrations, together with $T_{S P}$ and $T_{N}$ determined by susceptibility (in a column labelled $\chi$ ). The effective atomic displacement $\delta_{\text {eff }}$ at $T=T_{N}$ and magnetic moment

Table I. Summary of physical quantities characterizing SP properties of $\left(\mathrm{Cu}_{1-x} \mathrm{Mg}_{x}\right) \mathrm{GeO}_{3}$ obtained from the present neutron scattering data, except for the column designated by $(\chi)$ which is $T_{S P}$ determined previously

\begin{tabular}{|c|c|c|c|c|}
\hline \multirow[t]{2}{*}{$x$} & \multicolumn{2}{|c|}{$T_{S P}(\mathrm{~K})$} & \multirow[t]{2}{*}{$\Delta T(\mathrm{~K})$} & \multirow[t]{2}{*}{$\delta_{e f f}$} \\
\hline & $\chi$ & neutron & & \\
\hline 0.0 & - & 13.3 & & 1.00 \\
\hline 0.017 & 10.8 & 10.7 & 0.5 & $0.66(2)$ \\
\hline 0.026 & - & 8.8 & 0.9 & $0.57(5)$ \\
\hline 0.028 & - & 8.5 & 0.7 & $0.45(2)$ \\
\hline 0.032 & - & 8.0 & 1.1 & $0.28(1)$ \\
\hline
\end{tabular}
by magnetic susceptibility measurements[ref. 9]. See details in the text.

$\mu_{\text {eff }}$ at $T \sim 0 \mathrm{~K}$ are also listed. $T_{N}$ values determined using Eq. (1) are in good agreement with those obtained from magnetic susceptibility data.

\subsection{Order parameter}

We now closely examine the two sets of order parameters, $I_{S P} \propto \delta_{\text {eff }}^{2}$ and $I_{A F} \propto \mu_{e f f}^{2}$, over a wide $x$ range in order to construct the phase diagram from the neutron-scattering measurements. To scale the order parameters properly, a series of superlattice and fundamental reflections were collected for all the samples in the $(0 k l)$ and $(h k h)$ zones for the AF and SP reflections, respectively. The results are summarized in Figs. 4 and 5. Note that the SP intensity significantly decreases as $x$ increases.

A very simple yet clear demonstration of the phase boundary at $x_{c}$ is a plot of the intensity data as a function of $x$ at a given temperature. As shown in Fig. 6, the $x$ dependence of $\mu_{\text {eff }}^{2}$ at $1.5 \mathrm{~K}$ and $3.0 \mathrm{~K}$ as well as $\delta_{\text {eff }}^{2}$ at $1.3 \mathrm{~K}$ and $7.0 \mathrm{~K}$ clearly indicate that there exists a critical concentration between $x=0.026$ and 0.028 , though we have rather small number of $x$ points; only five. The 
Table II. Summary of physical quantities characterizing AF properties of $\left(\mathrm{Cu}_{1-x} \mathrm{Mg}_{x}\right) \mathrm{GeO}_{3}$ obtained from the present neutron scattering data, except for the column designated by $(\chi)$ which is $T_{N}$ determined previously by magnetic susceptibility measurements[ref. 9]. See details in the text.

\begin{tabular}{ccccc}
\hline \multicolumn{3}{c}{$T_{N}(\mathrm{~K})$} & $\Delta T(\mathrm{~K})$ & $\mu_{\text {eff }}\left(\mu_{\mathrm{B}}\right)$ \\
& $\chi$ & neutron & \\
\hline 0.017 & 2.7 & 2.4 & 0.3 & $0.17(1)$ \\
0.026 & $4.0,3.4$ & 3.2 & 0.4 & $0.28(1)$ \\
0.028 & 4.2 & 3.9 & 0.3 & $0.36(2)$ \\
0.032 & 4.2 & 4.2 & 0.2 & $0.34(1)$ \\
0.041 & 4.5 & 4.3 & 0.2 & $0.24(2)$ \\
0.082 & 2.1 & 1.9 & 0.2 & $0.16(1)$ \\
\hline
\end{tabular}

Fig. 4. Temperature dependence of superlattice intensity $I_{S P}$, which is proportional to $\delta_{e f f}^{2}$, for various concentrations. The values are normalized by $I_{S P}$ at $T=0 \mathrm{~K}$ for a pure system $(x=0)$. Solid curves are guides for the eye. For $x>x_{c}, \delta_{e f f}^{2}(T=0)$ becomes nearly zero while for $x<x_{c}$ it remains finite.

Fig. 5. Temperature dependence of the magnetic superlattice intensity $I_{A F}$, proportional to $\mu_{e f f}^{2}$, for various concentrations. The values are normalized by the $\left(\begin{array}{lll}0 & 2 & 1\end{array}\right)$ fundamental reflection. Solid curves are guides for the eye.

critical concentration can be also seen in the $x$ dependence of $T_{N}$. These plots lead to a simple picture that the AF order parameter is suddenly enhanced at $x_{c}$, accompanied by a precipitous drop of the lattice dimerization. However, $\delta$ remains finite at finite temperature and approaches zero only in the low temperature limit. (See Fig. 4.)

The present neutron-scattering measurements have confirmed the existence of a compositional

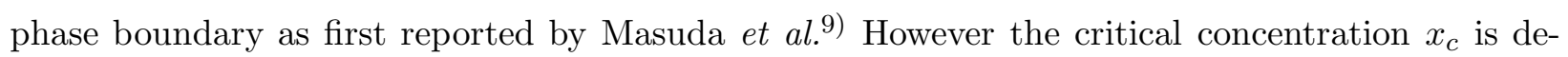
termined to be $0.027 \pm 0.001$, which is somewhat larger than $x_{c}=0.023$ previously reported from susceptibility measurements.9) This difference is most likely caused by coexistence of two phases in 
Fig. 6. Concentration dependence of (a) $T_{N}$, (b) $\mu_{\text {eff }}^{2}$ at $T=1.5,3.0 \mathrm{~K}$, and (c) $\delta_{\text {eff }}^{2}$ at $T=1.3,7.0 \mathrm{~K}$. All data clearly show abrupt changes at the critical concentration $x_{c}=0.027 \pm 0.001$, evidence for the compositional phase boundary at $x_{c}$.

Fig. 7. (a) The peak profile of $I_{S P}\left(\frac{3}{2}, 1, \frac{3}{2}\right)$ plotted with closed circles measured in the direction of $[0 k 0]$ at $T=5 \mathrm{~K}$ for $x=0.032>x_{c}$ is significantly broader than the experimental resolution represented with open circles. (b) The inverse correlation length plotted against temperature.

the vicinity of $x_{c}$. The order parameter $I_{A F}$ measured by neutron scattering is dominated by the majority phase, while susceptibility data may show two anomalies corresponding to the majority and minority phases if the latter has enough volume. The susceptibility data for $x=0.023$ in Fig. 1 shows two AF anomalies. The anomaly at higher temperature, however, exhibits a tiny change while the other shows a large drop, indicating that the lower $T_{N}$ is still dominant at $x=0.023$. Therefore we believe that the actual compositional phase boundary is located higher than $x=0.023$, namely $x_{c}=0.027 \pm 0.001$ as determined from the present neutron-scattering study.

\subsection{Line-broadening}

Throughout the whole range of concentrations studied, the AF phase shows long-range ordering; the superlattice peaks are almost resolution-limited. On the other hand, the SP phase shows a slight line-broadening at high concentrations beyond $x_{c}$. Figure 7(a) shows a typical peak profile of SP reflection measured around $\left(\frac{3}{2} 1 \frac{3}{2}\right)$ for $x=0.032$ at $T=5 \mathrm{~K}$. The instrumental resolution was experimentally determined using the $\left(\begin{array}{lll}3 & 2 & 3\end{array}\right)$ fundamental peak employing $\frac{\lambda}{2}$ incident neutrons. It is clear that the SP reflection shows a significant broadening along the $[0 k 0]$ direction. Note that such a broadening is not obvious in the $\left[\begin{array}{lll}h & 0 & h\end{array}\right]$ direction, indicating anisotropic correlation lengths; the correlation length $\xi_{[h 0 h]}$ is at least 1.5 times longer than $\xi_{[0 k 0]}$.

Such a remarkable broadening along $\left[\begin{array}{lll}0 & k & 0\end{array}\right]$ is observable in a wide temperature range as shown in Fig. 7(b), where the intrinsic line-width, i.e. the inverse correlation length $\kappa$ is plotted as a function of temperature. Note that the development of a long-range SP order is considerably suppressed below $T_{N}$, which is consistent with the decrease of $I_{S P}$ in the same temperature range. At present the data on line widths are limited. They seem to suggest that the correlation length $\xi$ doesn't have 
Fig. 8. The $T-x$ phase diagram of $\left(\mathrm{Cu}_{1-x} \mathrm{Mg}_{x}\right) \mathrm{GeO}_{3}$ based on the present neutron data. The shaded region represents a short-range ordered SP state newly observed. The critical concentration is nearly independent of temperature as shown with an obliquely-dashed line.

an anomaly at the critical concentration $x_{c}$, but changes continuously 14 A similar line broadening was already reported by Martin et al. concerning inhomogeneity in Zn-doped samples, which makes quantitative discussions difficult.

\section{$\S 4 . \quad$ DISCUSSION}

As shown in the $T-x$ phase diagram of $\left(\mathrm{Cu}_{1-x} \mathrm{Mg}_{x}\right) \mathrm{GeO}_{3}$ constructed by present neutronscattering study (Fig. 8), one can clearly see the existence of a compositional phase boundary at the critical concentration $x_{c}$. Saitd 15 has theoretically studied impurity effects in a disordered spinPeierls system and suggested that the long-range SP order disappears above a critical concentration of impurity. The lattice dimerization $\delta$ extrapolated to $T=0 \mathrm{~K}$ (See Fig. 6(c)) as a function of concentration $x$ is consistent with the theoretical prediction. At present, the theory discusses only the ground state properties. We believe that the "intermediate" phase indicated by a shaded region, where the short-range ordered SP state exists, will be also clarified by extending the theory to finite temperatures.

Figure 9 shows a relation between $T_{N}$ and the effective magnetic moment $\mu_{e f f}$ of $\left(\mathrm{Cu}_{1-x} \mathrm{Mg}_{x}\right) \mathrm{GeO}_{3}$ at the low temperature limit. Data for $\mathrm{Zn}$-doped and Si-doped $\mathrm{CuGeO}_{3}$ are

Fig. 9. The effective magnetic moment $\mu_{\text {eff }}$ of $\left(\mathrm{Cu}_{1-x} \mathrm{Mg}_{x}\right) \mathrm{GeO}_{3}$ evaluated at the low temperature limit $(T=1.3 \mathrm{~K})$ plotted against $T_{N}$ (closed circles). Previously reported data on $\left(\mathrm{Cu}_{1-x} \mathrm{Zn}_{x}\right) \mathrm{GeO}_{3}[\mathrm{ref} .7]$ and $\mathrm{Cu}\left(\mathrm{Ge}_{1-x} \mathrm{Si}_{x}\right) \mathrm{O}_{3}[$ ref. 11, 12] are also shown.

also shown. We notice that $\mu_{e f f}$ increases linearly as $T_{N}$ increases, though the gradient seems to depend upon dopant. Note that the anomaly of $\mu_{e f f}$ at $x_{c}$ is not very clear in this plot. This is because the anomaly in $\mu_{e f f}$ is accompanied by the jump of $T_{N}$ at $x_{c}$.

It is now clear that there exist two types of AF state separated by the compositional phase boundary at $x_{c}$. However, the origin of such a difference is still not completely understood. It is interesting to study how the difference between the dimerized (D-AF) and undimerized AF (U-AF) 
states appears in the magnetic excitations. In addition to performing a comprehensive study of spin waves at different $x$, we plan to investigate how the structural dynamics, i.e. phonon and phason, affect the magnetic excitations by employing polarized neutrons.

In conclusion, the present neutron-scattering study of $\left(\mathrm{Cu}_{1-x} \mathrm{Mg}_{x}\right) \mathrm{GeO}_{3}$ with a wide range of $x$ has revealed that the order parameters such as the lattice dimerization $\delta$ and the AF magnetic moment show a sudden change at the critical concentration $x_{c}=0.027 \pm 0.001$. This indicates the existence of a compositional phase boundary between two distinct AF phases. We also found that there exists an intermediate phase with a short-range SP order at $x>x_{c}$ in a certain finite temperature range.

\section{Acknowledgement}

We acknowledge M. Saito and H. Fukuyama for fruitful discussions. We thank S. Watanabe for technical support on our experiments. Two of authors (H.N. and T.M.) acknowledge support by the Japan Society for the Promotion of Science for Young Scientists. This study was supported in part by the U.S.-Japan Cooperative Program on Neutron Scattering between USDOE and MONBUSHO and by NEDO (New Energy and Industrial Technology Development Organization) International Joint Research Grant.

[1] M. Hase, I. Terasaki, and K. Uchinokura, Phys. Rev. Lett. 70 (1993) 3651.

[2] L. P. Regnault, J. P. Renard, G. Dhalenne and A. Revcolevschi, Europhys. Lett. 32 (1995) 579.

[3] H. Fukuyama, T. Tanimoto, and M. Saito, J. Phys. Soc. Jpn. 65 (1996) 1182.

[4] K. M. Kojima, Y. Fudamote, M. Larkin, G. M. Luke, J. Merrin, B. Nachumi, Y. J. Uemura, M. Hase, Y. Sasago, K. Uchinokura, Y. Ajiro, A. Revcolevshi, and J. -P. Renard, Phys. Rev. Lett. 79 (1997) 503.

[5] K. Manabe, H. Ishimote, N. Koide, Y. Sasago, and K. Uchinokura, Phys. Rev. B58 (1998) R575.

[6] Y. Sasago, N. Koide, K. Uchinokura, M. C. Martin, M. Hase, K. Hirota, and G. Shirane, Phys. Rev. B54 (1996) R6835.

[7] M. C. Martin, M. Hase, K. Hirota, G. Shirane, Y. Sasago, N. Koide, and K. Uchinokura, Phys. Rev. B56 (1997) 3173.

[8] Y. Ajiro, T. Asano, F. Msui, M. Mekata H. Aruga-Katori, T. Goto, H. Kikuchi , Phys. Rev. B51 (1995) 9399.

[9] T. Masuda, A. Fujioka, Y. Uchiyama, I. Tsukada, and K. Uchinokura, Phys. Rev. Lett. 80 (1998) 4566.

[10] J. P. Schoeffel, J. P. Pouget, G. Dhalenne, and A. Revcolevschi, Phys. Rev. B53 (1996) 14971.

[11] K. Hirota, M. Hase, J. Akimitsu, T. Masuda, K. Uchinokura, and G. Shirane, J. Phys. Soc. Jpn. 67 (1998) 645.

[12] S. Katano, O. Fujita, J. Akimitsu, M. Nishi, K. Kakurai, and Y. Fujii, Phys. Rev. B57 (1998) 10280.

[13] K. Hirota, G. Shirane, Q. J. Harris, Q. Feng, R. J. Birgeneau, M. Hase, and K. Uchinokura, Phys. Rev. B52 (1995) 15412.

[14] H. Nakao at el., unpublished data.

[15] M. Saito, J. Phys. Soc. Jpn. 67 (1998) 2477. 

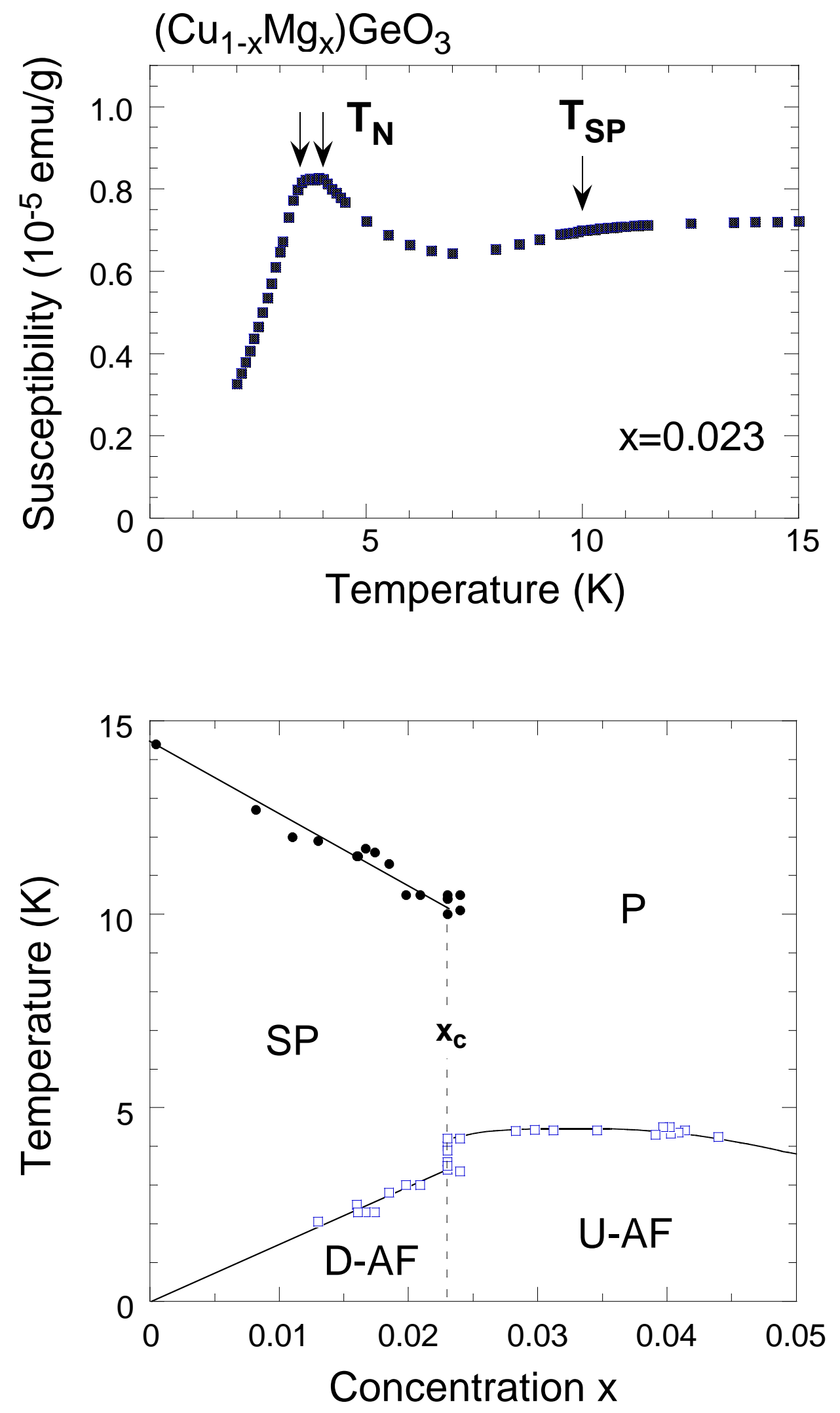

Fig.1 H. Nakao et al. 


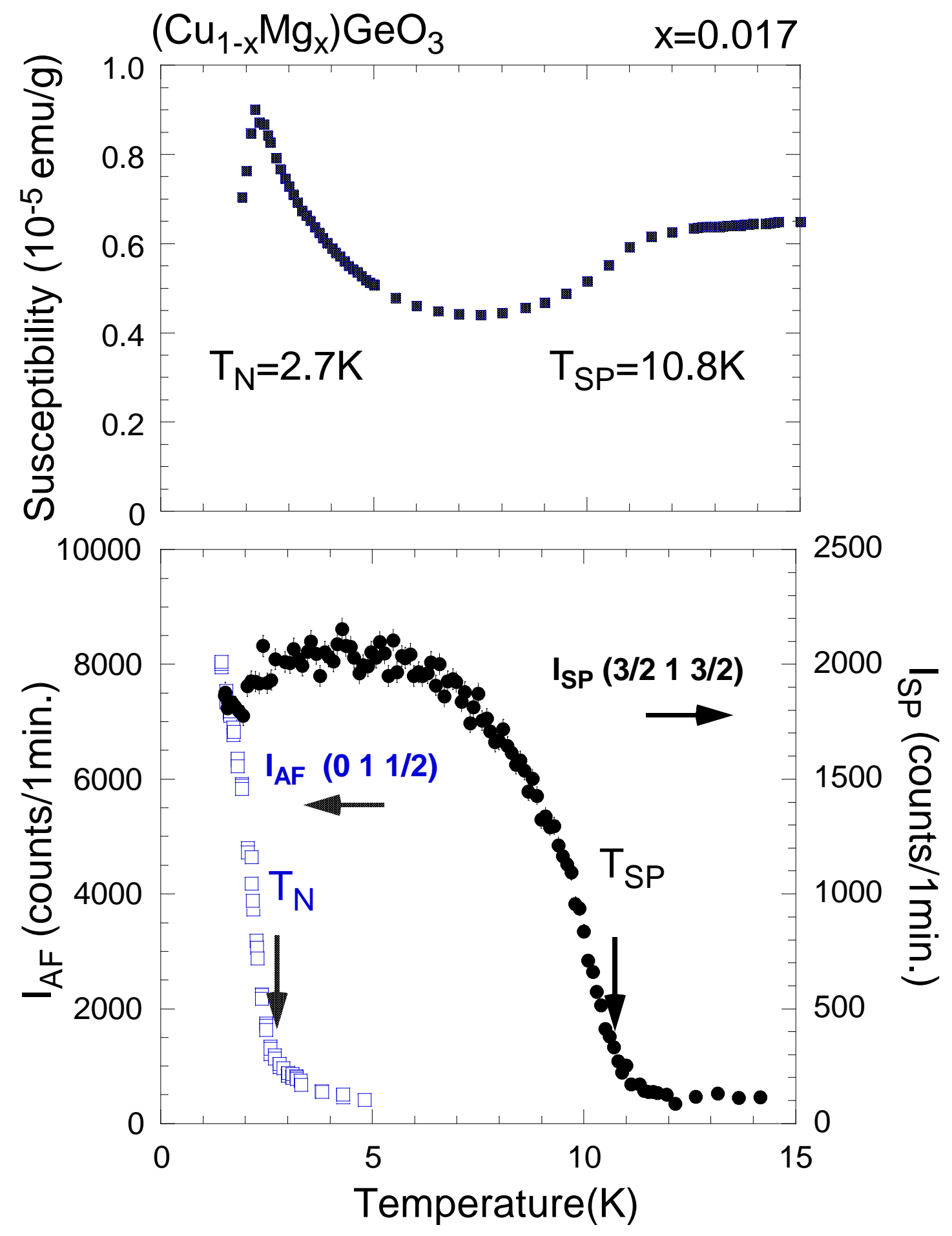

Fig.2 H. Nakao et al. 


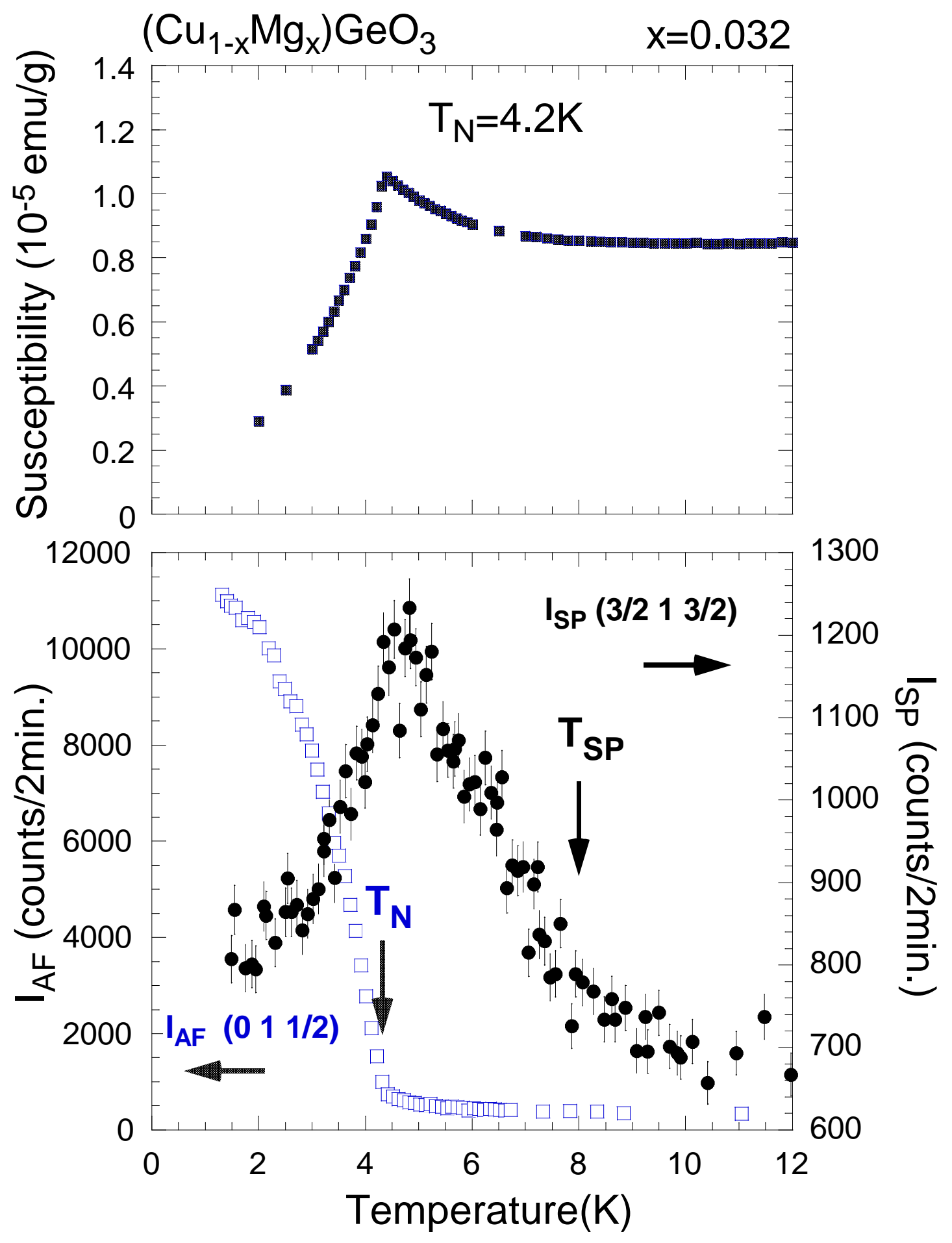

Fig.3 H. Nakao et al. 


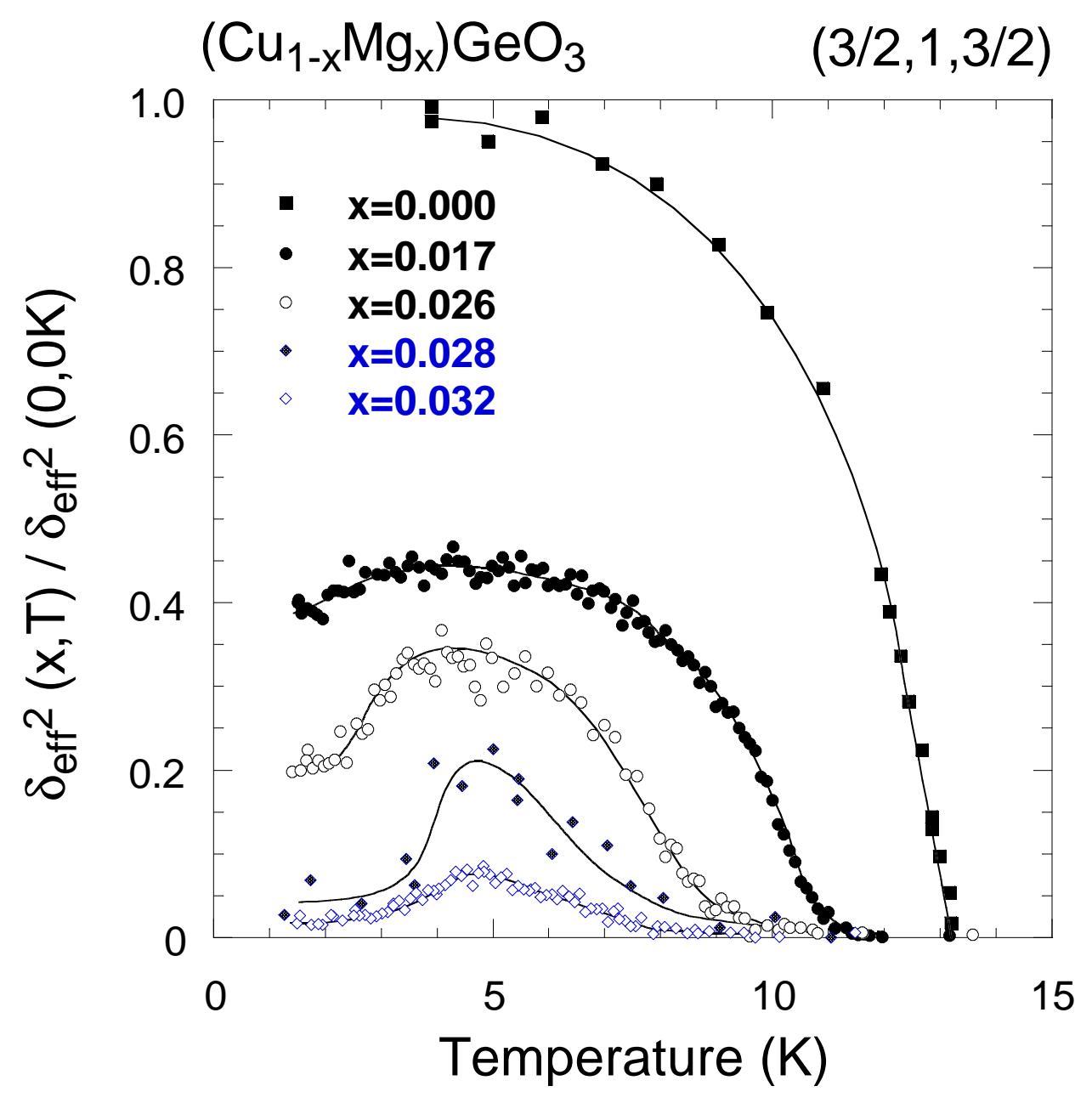

Fig.4 H. Nakao et al. 


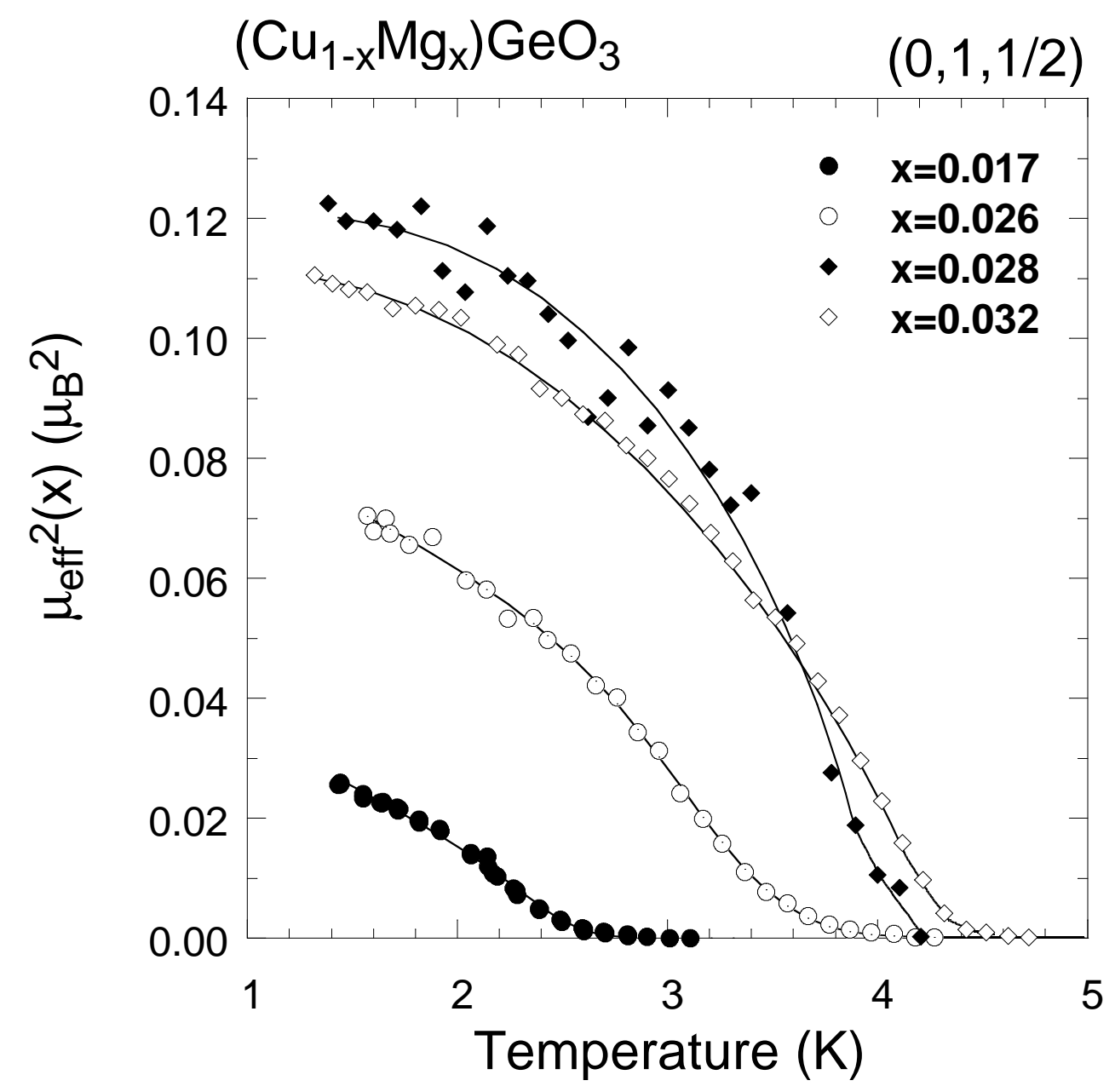

Fig.5 H. Nakao et al. 

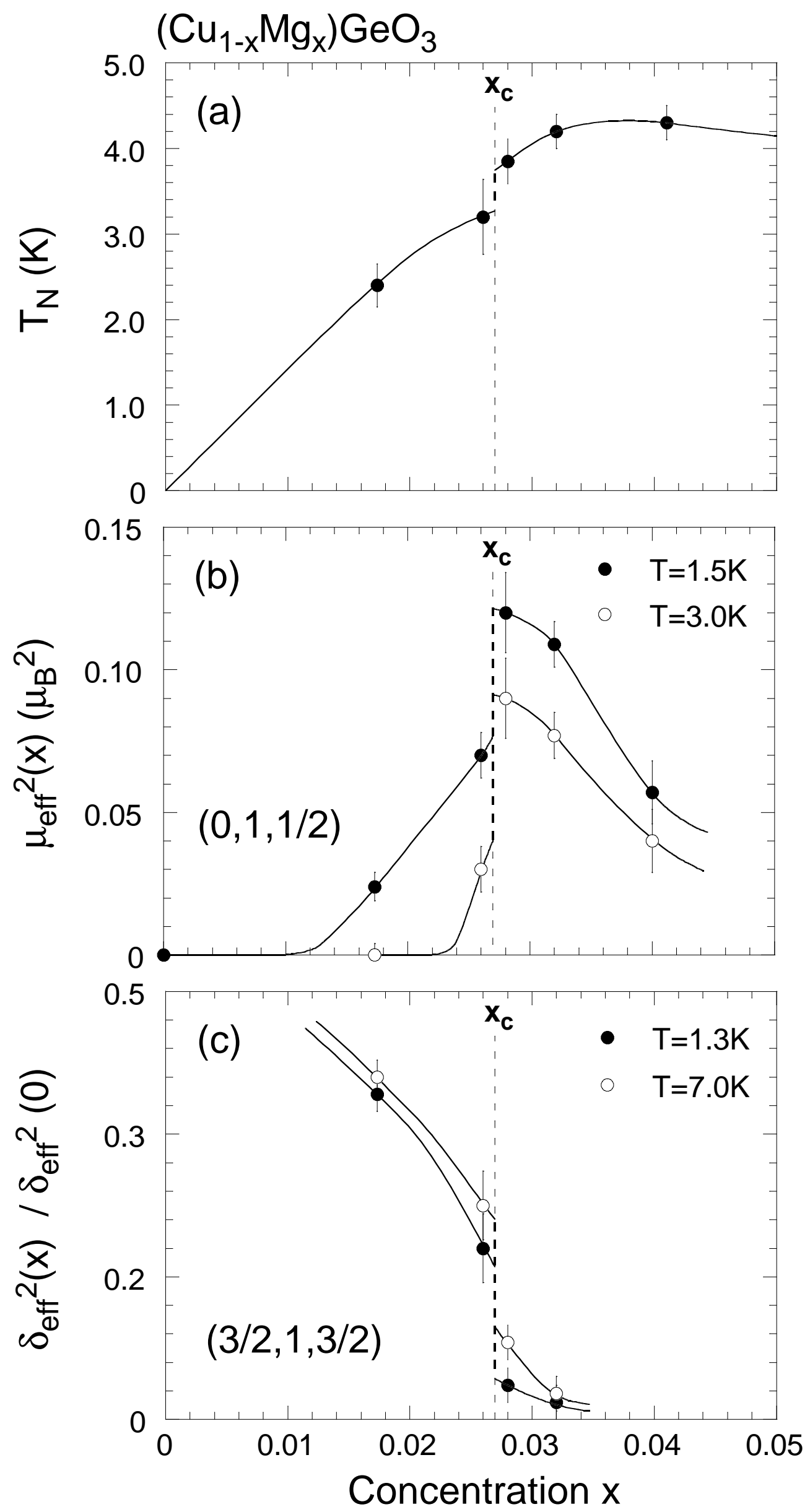

Fig.6 H. Nakao et al. 

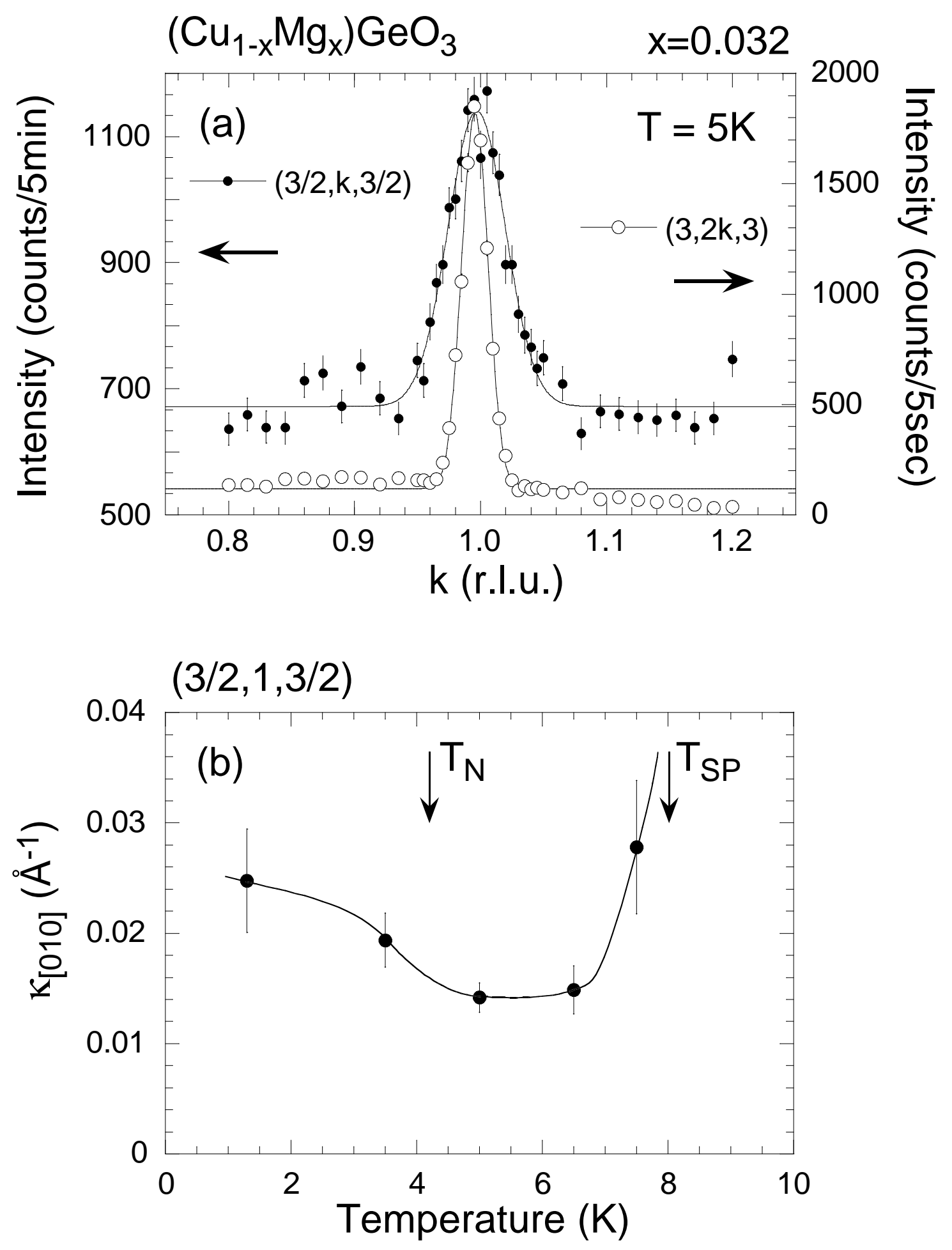

Fig.7 H. Nakao et al. 


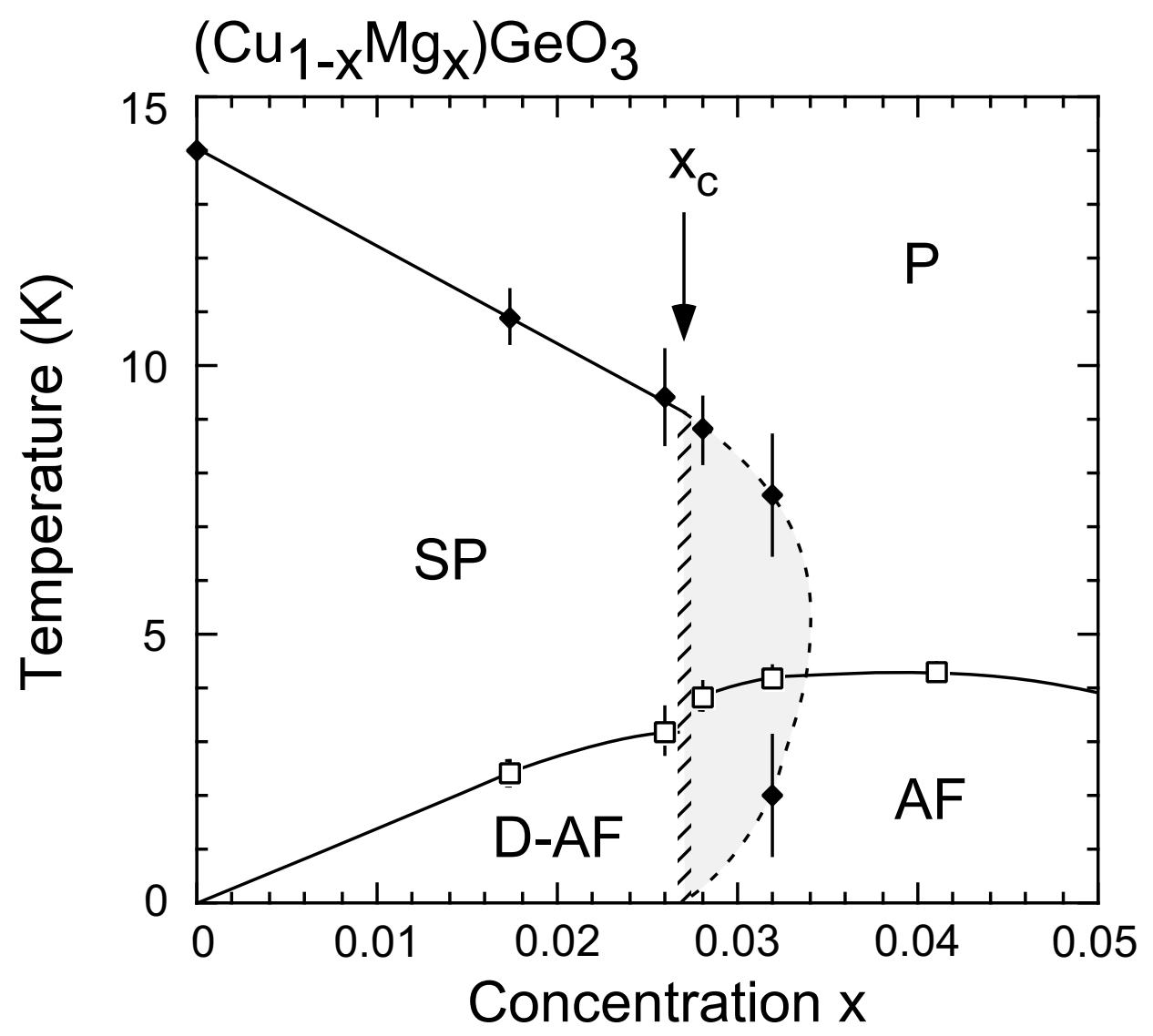

Fig. 8 H.Nakao et al. 


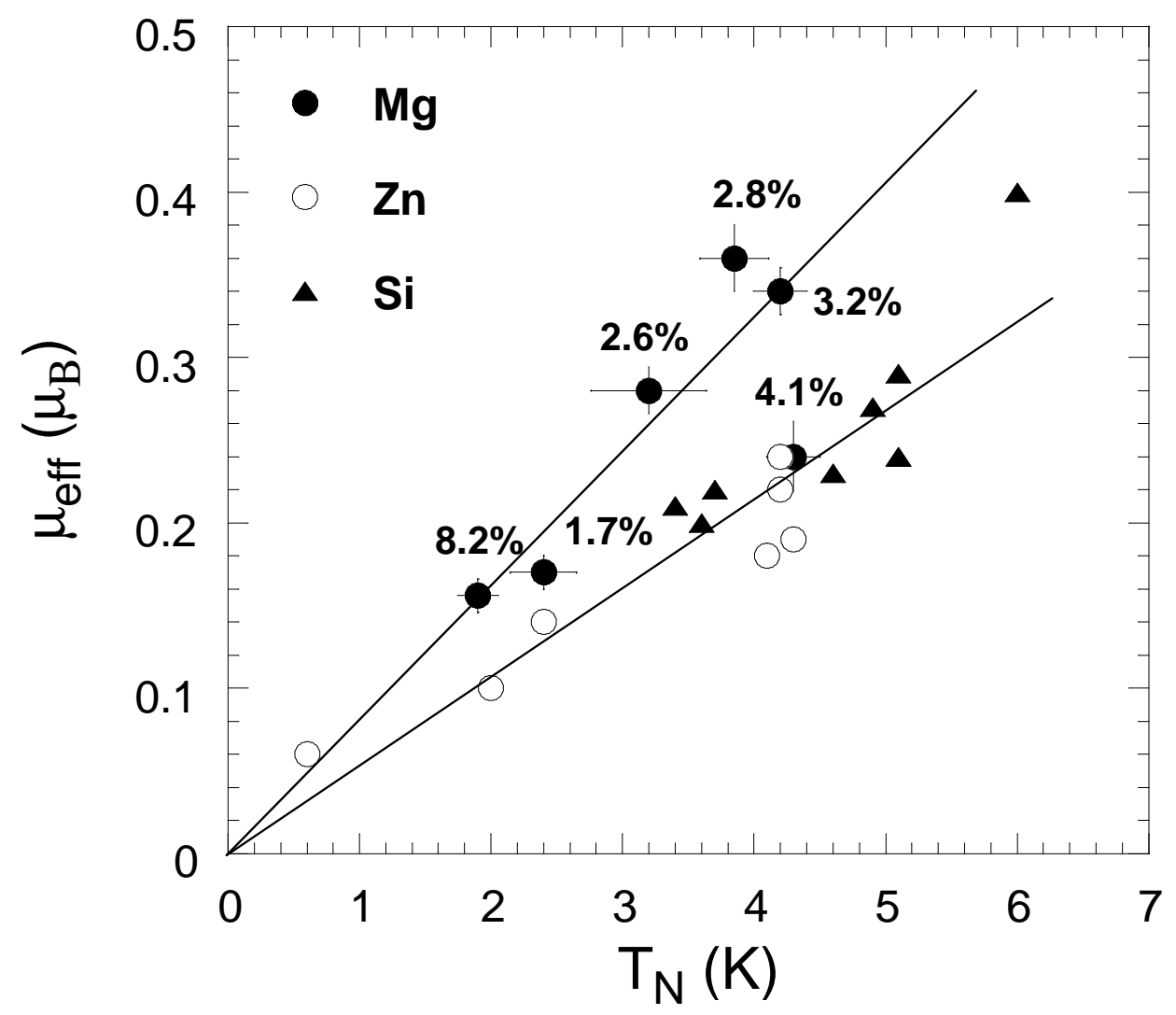

Fig.9 H. Nakao et al. 